\title{
Group cognitive-behavioral therapy in insomnia: a cross-sectional case-controlled study
}

\author{
This article was published in the following Dove Press journal: \\ Neuropsychiatric Disease and Treatment \\ Number of times this article has been viewed
}

\author{
Hongjing Mao',* \\ Yutian $\mathrm{ji}^{2}$ ** \\ You $\mathrm{Xu}{ }^{\prime}$ \\ Guangzheng Tang' \\ Zhenghe Yu' \\ Lianlian $X u^{\prime}$ \\ Chanchan Shen ${ }^{2}$ \\ Wei Wang ${ }^{1,2}$ \\ 'Department of Psychosomatic \\ Disorders, The Seventh People's \\ Hospital, Mental Health Center, \\ ${ }^{2}$ Department of Clinical Psychology \\ and Psychiatry, School of Public \\ Health, Zhejiang University College \\ of Medicine, Hangzhou, Zhejiang, \\ People's Republic of China \\ *These authors contributed equally \\ to this work
}

Background: Group cognitive-behavioral therapy (GCBT) might meet the considerable treatment demand of insomnia, but its effectiveness needs to be addressed.

Participants: This study recruited 27 insomnia patients treated with 16-weeks of zolpidem (zolpidem group), 26 patients treated with 4-weeks of zolpidem and also treated with 12-weeks of GCBT (GCBT group), and 31 healthy control volunteers.

Methods: Before treatment and 16 weeks after intervention, participants were evaluated using the Patient Health Questionnaires (Patient Health Questionnaire-9 [PHQ-9] and Patient Health Questionnaire-15 [PHQ-15]), the Dysfunctional Beliefs and Attitudes about Sleep-16 (DBAS-16), and the Pittsburgh Sleep Quality Index (PSQI).

Results: Compared to the zolpidem and healthy control groups, the scale scores of PHQ-9, PHQ-15, DBAS-16 and PSQI were significantly reduced after intervention in the GCBT group. Regarding the score changes, there were correlations between PSQI, DBAS-16, PHQ-9, and PHQ-15 scales in the zolpidem group, but there were limited correlations between PSQI and some DBAS-16 scales in the GCBT group.

Conclusion: Our results indicate that GCBT is effective to treat insomnia by improving sleep quality and reducing emotional and somatic disturbances; thus, the study supports the advocacy of applying group psychotherapy to the disorder.

Keywords: cognitive-behavioral therapy, group psychotherapy, insomnia

\section{Introduction}

Insomnia is the most common form of sleep disruption, which principally comprises sustained difficulties in initiating or maintaining sleep. Approximately one-third of the general population have experienced lifelong symptoms of insomnia, and $\sim 6 \%$ adults meet the diagnostic criteria for insomnia disorders. ${ }^{1}$ Insomnia has its natural remission rate of $54 \%$, and $27 \%$ patients have experienced a relapse, ${ }^{2}$ however, it contributes to various psychosomatic disorders. ${ }^{3-5}$

Pharmacotherapy for insomnia has produced reliable improvements in sleep but is mainly intended for short-term use, ${ }^{6,7}$ due to its possible addiction, withdrawal and tolerance syndromes and due to its daytime dysfunctional symptoms. ${ }^{8,9}$ The short-term value of pharmacotherapy is contrasted by that of psychotherapy applied in insomnia, for instance, the effect of cognitive-behavioral therapy (CBT). CBT is aimed at changing sleep-related cognitions such as the faulty beliefs and attitudes, ${ }^{10}$ to correct the maladaptive sleep habits, and to reduce autonomic and cognitive arousal levels. ${ }^{11}$ The therapy has lasting improvements in subjective sleep quality among $70 \%-80 \%$ of patients and supports the hypnotic reduction and withdrawal. ${ }^{12}$ It also leads to the modest relief of both physical and psychological symptoms of insomnia, such as pain, depression, and anxiety. ${ }^{13-15}$
Correspondence: Wei Wang Department of Clinical Psychology and Psychiatry, School of Public Health, Zhejiang University College of Medicine, Yuhangtang Road 866, Hangzhou,

Zhejiang 310058 , People's Republic of China

Tel +86 57I 88208188

Email drwangwei@zju.edu.cn 
The abovementioned studies were concerning the CBT delivered to individual patients. The CBT practice itself is complicated, time-consuming, and expensive compared to other therapies. ${ }^{16}$ Furthermore, the contradiction of high demand of CBT and its short supply hampers its dissemination in treating insomnia. ${ }^{16,17} \mathrm{Up}$ to date, pharmacotherapy remains one of the common treatment necessities for insomnia. In the meantime, other derivative CBT methods have been developed and widely accepted in clinics, for instance, online CBT and group CBT (GCBT), both of which have proven effective and time-saving when treating patients with insomnia. ${ }^{18,19}$ While in populations who are not comfortable with Internet use or are living in the regions with poor broadband Internet access, it is hard for the online CBT to disseminate. ${ }^{20}$ In this case, the GCBT might have a broader applicability in treating the disorder.

Although GCBT has proven effective in treating insomnia, there are actually many patients who have been treated with, but benefited less, from medication; thus, there is a consistent need for launching both pharmaco- and psychotherapies for this population. ${ }^{9,21}$ Similarly in China, it is easier to apply both therapies rather than the psychotherapy alone to insomnia patients. The question however still remains how much insomnia patients, actually those treated with medication, would benefit from the GCBT.

Thereupon, we enrolled a group of healthy volunteers and randomly enrolled two groups of insomnia patients treated with a routine hypnotic medication (zolpidem), one group of which also received GCBT. The GCBT we administered was a modification of Morin's therapy for a group delivery, which was aiming at the restructuring of dysfunctional beliefs and attitudes about sleep and at the reshaping of sleep patterns of patients. ${ }^{22}$ We have hypothesized that GCBT improves sleep quality (its latency, duration, and efficiency) and decreases sleep disturbance and daytime physical and psychological dysfunctions of insomnia patients. In addition, we have explored the potential relationships between the dysfunctional beliefs and attitudes, somatic and emotional states, and sleep conditions. In three groups, at two time points of before (baseline) and after the treatment/intervention, or waiting period (for healthy volunteers), we administered the Dysfunctional Beliefs and Attitudes about Sleep-16 (DBAS-16) to measure the dysfunctional beliefs and attitudes about sleep, ${ }^{23}$ the Pittsburgh Sleep Quality Index (PSQI) to measure the sleep quality, ${ }^{24}$ the Patient Health Questionnaire-9 (PHQ-9) to measure the psychological symptoms relating to sleep, ${ }^{25}$ and the Patient Health Questionnaire-15 (PHQ-15) to measure the physical symptoms relating to sleep. ${ }^{26}$

\section{Methods}

\section{Participants}

A total of 53 patients suffering from insomnia were invited to participate in the current study. They were diagnosed according to the Diagnostic and Statistical Manual of Mental Disorders, Fifth Edition, criteria ${ }^{27}$ by two experienced psychiatrists (HM and WW, also coauthors of the present article) ${ }^{27}$ Similar to the clinical description, ${ }^{27}$ almost all patients had received additional diagnoses of depression or anxiety or both, but they were secondary to insomnia. They were randomly divided into two groups: 27 patients (zolpidem group; seven males and 20 females; mean age: 45.04 years with $7.79 \mathrm{SD}$; age range: $26-67$ years) received only zolpidem (10 mg/night; Sanofi S.A., Paris, France) for 16 weeks and 26 patients (GCBT group; four males and 22 females; mean age: $43.27 \pm 12.03$ years; age range: 25-65 years) received both zolpidem $(10 \mathrm{mg} / \mathrm{night})$ and GCBT for the beginning 4 weeks of study and afterward were asked to stop zolpidem (but they might take it when needed) and received GCBT continuously for the next 12 weeks (altogether 16 weeks). The insomnia histories of these patients varied from 7 months to 25 years, and there was no significant difference in histories between the two patient groups $(t[1,51]=2.82, p=0.24)$. No patient had brain lesions according to recent computerized tomography or magnetic resonance imaging scans or had suffered from alcohol abuse or tobacco use or other types of psychiatric disorder. In all, 31 healthy volunteers (eight males and 23 females; mean age: $43.56 \pm 9.92$ years; age range: $24-62$ years) were recruited from students, hospital staff, and paid volunteers from the general population. They were not suffering or had not suffered from any psychiatric problems, including alcohol or tobacco abuse, or other types of personality disorder, assessed through a semi-structured clinical interview, also by two experienced psychiatrists. All 84 participants had received $>10$ years of education. No significant difference was found between the three groups regarding age $(F[2,81]=0.47, p=0.63$, mean square effect $[\mathrm{MSE}]=47.44)$, gender $\left(\chi^{2}=1.13, p=0.57\right)$, or education level $(F[2,78]=1.57$, $p=0.21, \mathrm{MSE}=2.06$ ). The study protocol was approved by the Mental Health Center of The Seventh People's Hospital, and all participants provided their written informed consent to partake in the current study.

\section{Group cognitive-behavioral therapy}

The healthy volunteers received no medication or psychotherapy during the study period. The leading author (HM) and colleagues delivered both GCBT and zolpidem therapies 
to patients. The GCBT program was structured into eight sessions that were held fortnightly (every 2 weeks), each session lasting for 2 hours. Six to eight participants formed a small group to attend the sessions. Each session had a particular focus, and the therapist (HM) and nurses delivered some specific techniques or skills. For standardizing GCBT procedure, all steps strictly followed a written manual (Table S1). Briefly, the sleep hygiene education emphasized the intra- and interindividual differences regarding the sleep demands at different ages. The psychoeducation included sleep-related cognition, behavior, and emotion and their managements. The education courses included the comparisons between clinically defined and self-diagnosed insomnia, varied causes of insomnia (food, alcohol, stimulants, medication for other purposes), and management of inappropriate behaviors relating to sleep feedbacks (sleep and bed, bedtime restriction, and food-water-substance intake), some of which were controlled/assessed through electroencephalogram and other biotechniques and mood (anxiety and depression) controls. The psychoeducation helped patients to get rid of misunderstandings about insomnia, to behave appropriately, to neutralize fluctuating emotions, to (self-) check the results, and to reduce insomnia relapse. For bedtime control, participants were asked to keep awake during the daytime, without any napping; before going to bed, they were asked to avoid using cigarettes, wine, caffeine, or other substances and to avoid participating in exciting or sentimental activities; when lying in bed, they were asked to avoid spending much time talking, telephoning, Internet using, or thinking (ie, the function of bed is only for sleeping; an individual might go to bed only when he/she is sleepy, otherwise, gets up and does the things he is busy with).

\section{Questionnaires}

Before (baseline) and after the treatment/intervention, or waiting period (for healthy volunteers), participants were asked to complete the following four questionnaires in a quiet room under supervision of a member of the current author group.

\section{PHQ-9}

PHQ-925 is a screening instrument with nine items to measure the depressive mood relating to sleep. For each item, patients were asked to assess how much they were bothered by the symptoms over the last 2 weeks using a Likert scale: 0 - not at all, 1 - several days, $2->7$ days, and 3 - nearly every day. The higher score indicates more severe depression. Its internal reliability (the Cronbach's alpha) was 0.80 in the present study.

\section{PHQ- I5}

PHQ-15 $5^{26}$ comprises 15 items measuring somatic symptoms relating to sleep, which account for $>90 \%$ of the physical or somatic complaints reported in the outpatient setting. Each symptom is scored from 0 (not bothered at all) to 2 (bothered a lot), with a higher score indicating more severe somatic symptoms. Its internal reliability was 0.89 in the present study.

\section{DBAS- 16}

DBAS- $16^{23}$ is a 16 -item self-report questionnaire designed to identify and assess various sleep-related cognitions, consisting of four subscales: consequence of insomnia (five items), worrying about sleep (six items), sleep expectations (two items), and medication attribution (three items). Each item (statement) is rated using a Likert scale, from 1 (strongly disagree) to 5 (strongly agree), with a higher score indicating more severe dysfunctional beliefs and attitudes. Its internal reliability was 0.80 in the present study.

\section{PSQI}

$\mathrm{PSQI}^{24}$ typically measures the subjective sleep quality and disturbances during the previous month. The questionnaire consists of 19 questions of self-assessment and five questions rated by the bed partner or roommate. The 19 items are grouped into seven scales: subjective sleep quality (one item), sleep latency (two items), sleep duration (one item), habitual sleep efficiency (three items), sleep disturbances (nine items), use of sleeping medication (one item), and daytime dysfunction (two items). Each item is rated using a Likert scale from 0 to 3, with a higher score indicating worse sleep quality. Its internal reliability was 0.70 in the present study.

\section{Statistical analyses}

The changes in the scale score of the four questionnaires were defined as their differences before and after the conditioned situation, for instance, intervention/treatment or waiting period (ie, baseline minus conditioned). Two-way analysis of variance (ANOVA) was applied to the baseline scores of seven PSQI or the five DBAS-16 scales and their changes in three groups of participants. One-way ANOVA was applied to the baseline scores of PHQ-9 and PHQ-15 and their changes in three groups of participants. Whenever a significant main effect was found, post hoc analysis by the least significant difference (LSD) test was used to evaluate between-group differences. When exploring the relationship between the insomnia history and the score changes in PHQ-9, PHQ-15, DBAS-16, and PSQI, we used the Pearson linear correlation test. A $p$-value $<0.05$ was considered to be significant. 


\section{Results}

\section{Baseline scale scores}

There were significant differences in scale scores of all measures between the three groups regarding PHQ-9 $(F[2,81]=15.70, p<0.001, \mathrm{MSE}=336.27)$, PHQ-15 $(F[2,81]=13.36, p<0.001, \operatorname{MSE}=144.38)$, DBAS-16 (group effect, $F[2,81]=9.59, p<0.001$, MSE $=255.27$; scale effect, $F[4,79]=498.93, p<0.001$, MSE $=3,994.14$; group X scale interaction effect, $F[8,75]=15.38, p<0.001$, MSE =123.09), and PSQI (group effect, $F[2,81]=72.62$, $p<0.001$, MSE $=109.23$; scale effect, $F[6,77]=13.29$, $p<0.001, \mathrm{MSE}=12.06$; group $\mathrm{X}$ scale interaction effect, $F[12,71]=5.62, p<0.001, \mathrm{MSE}=5.10)$. The healthy control group scored significantly lower on these scales than the two patient groups did (Table 1).

However, when the two patient groups were compared, there were no significant differences on the scale scores regarding PHQ-9 $(t=0.57, p=0.57)$, PHQ-15 $(t=0.92$, $p=0.36$ ), DBAS-16 (group effect, $F[1,51]=3.93, p=0.053$, MSE $=55.93$; scale effect, $F[4,48]=488.76, p<0.001$, $\mathrm{MSE}=3,468.70$; group $\mathrm{X}$ scale interaction effect, $F[4$, $48]=0.502, p=0.64, \mathrm{MSE}=3.56$ ), or PSQI (group effect, $F[1,51]=0.15, p=0.07, \mathrm{MSE}=0.32$; scale effect, $F[6,46]=13.80$, $p<0.001$, MSE $=18.48$; group $\mathrm{X}$ scale interaction effect, $F[6,46]=0.42, p=0.78$, MSE $=0.56$; Table 1$)$.

Table I Scale scores of the PHQ-9, PHQ-15, DBAS-16, and PSQI questionnaires from the GCBT $(n=26)$, zolpidem $(n=27)$, and healthy control $(n=31)$ groups before intervention

\begin{tabular}{llll}
\hline Scale & GCBT & Zolpidem & $\begin{array}{l}\text { Healthy } \\
\text { controls }\end{array}$ \\
\hline PHQ-9 & $7.31 \pm 5.88^{\mathrm{a}}$ & $6.41 \pm 5.54^{\mathrm{a}}$ & $1.03 \pm 1.56$ \\
PHQ-15 & $5.73 \pm 4.40^{\mathrm{a}}$ & $4.78 \pm 3.00^{\mathrm{a}}$ & $1.48 \pm 2.29$ \\
DBAS-16 & & & \\
Consequence of insomnia & $18.42 \pm 3.38^{\mathrm{a}}$ & $16.74 \pm 2.75^{\mathrm{a}}$ & $14.58 \pm 4.96$ \\
Worrying about sleep & $22.46 \pm 4.38^{\mathrm{a}}$ & $21.44 \pm 3.40^{\mathrm{a}}$ & $15.32 \pm 5.75$ \\
Sleep expectations & $7.42 \pm 1.79$ & $6.67 \pm 1.94$ & $4.48 \pm 2.28$ \\
Medication attribution & $8.65 \pm 1.96$ & $8.00 \pm 1.44$ & $7.84 \pm 2.84$ \\
PSQI & & & \\
Subjective sleep quality & $1.77 \pm 0.82^{\mathrm{a}}$ & $1.67 \pm 0.83^{\mathrm{a}}$ & $0.39 \pm 0.50$ \\
Sleep latency & $1.88 \pm 1.11^{\mathrm{a}}$ & $2.07 \pm 0.92^{\mathrm{a}}$ & $0.71 \pm 0.74$ \\
Sleep duration & $1.38 \pm 1.17^{\mathrm{a}}$ & $1.41 \pm 1.22^{\mathrm{a}}$ & $0.29 \pm 0.53$ \\
Habitual sleep efficiency & $1.62 \pm 1.36^{\mathrm{a}}$ & $1.67 \pm 1.24^{\mathrm{a}}$ & $0.13 \pm 0.43$ \\
Sleep disturbances & $1.81 \pm 0.75^{\mathrm{a}}$ & $1.63 \pm 0.49^{\mathrm{a}}$ & $0.94 \pm 0.44$ \\
Use of sleeping medication & $2.50 \pm 1.03^{\mathrm{a}}$ & $2.41 \pm 1.18^{\mathrm{a}}$ & $0.13 \pm 0.34$ \\
Daytime dysfunction & $1.12 \pm 0.82^{\mathrm{a}}$ & $0.81 \pm 0.79$ & $0.45 \pm 0.62$ \\
\hline
\end{tabular}

Notes: ${ }^{a} p<0.05$ vs healthy control group. Data presented as mean \pm SD. Abbreviations: DBAS-16, Dysfunctional Beliefs and Attitudes about Sleep-16; GCBT, group cognitive-behavioral therapy; PHQ-9, Patient Health Questionnaire-9; PHQ-15, Patient Health Questionnaire-15; PSQI, Pittsburgh Sleep Quality Index.

\section{Changes in scale score}

Three participant groups (healthy volunteers, zolpidem patients, and GCBT patients) had statistically different PHQ-9 score changes $(F[2,81]=3.30, p<0.05, \mathrm{MSE}=61.88)$, with those in the GCBT $(p<0.05,95 \%$ confidence interval $[\mathrm{CI}]=[0.34-4.92])$ and the zolpidem $(p<0.05,95 \% \mathrm{CI}=$ [0.12-4.65]) groups higher than that in the healthy controls. Three groups also had statistically different PHQ-15 score changes $(F[2,81]=3.14, p<0.05$, MSE $=22.72)$, with those in the GCBT group higher than those in healthy controls $(p<0.05,95 \% \mathrm{CI}=[0.36-3.21])$.

There were statistically significant differences on DBAS-16 score changes between three groups (group effect, $F[2,81]=17.93, p<0.001, \mathrm{MSE}=240.66$; scale effect, $F[4,79]=19.40, p<0.001, \mathrm{MSE}=108.22$; group $\mathrm{X}$ scale interaction effect, $F[8,75]=5.72, p<0.001$, MSE $=31.89$ ). The GCBT group reported significantly higher changes than the healthy $(p<0.001,95 \% \mathrm{CI}=[1.85-3.79])$ and zolpidem $(p<0.001,95 \% \mathrm{CI}=[1.20-3.20])$ groups did. In addition, there were statistically significant differences in PSQI score changes between the three groups (group effect, $F[2,81]=11.99, p<0.001, \mathrm{MSE}=14.87$; scale effect, $F[6$, $77]=1.84, p=0.11$, MSE $=1.25$; group $\mathrm{X}$ scale interaction effect, $F[12,71]=1.61, p=0.11$, MSE $=1.10)$. The GCBT group reported significantly higher changes than the healthy $(p<0.001,95 \% \mathrm{CI}=[0.32-0.77])$ and zolpidem $(p<0.01$, $95 \% \mathrm{CI}=[0.12-0.58])$ groups did (Table 2).

\section{Relationships between changes in scale score}

In the healthy control group, we did not find any correlations between score changes in PSQI and other inventory scales. There was no relationship between insomnia history and changes in the scale score of a given inventory in the two patient groups. In the zolpidem group, the score change in worrying about sleep of DBAS-16 was positively correlated with that of use of sleeping medication ( $\mathrm{n}=27, r=0.49, p<0.05$ ) and daytime dysfunction $(r=0.48, p<0.05)$ of PSQI; the score change in sleep expectations of DBAS-16 was positively correlated with that of sleep latency of PSQI $(r=0.42, p<0.05)$; the score change in PHQ-9 was positively correlated with that of habitual sleep efficiency $(r=0.45, p<0.05)$, sleep disturbances $(r=0.55, p<0.05)$, and daytime dysfunction $(r=0.46, p<0.05)$ of PSQI, and the score change in PHQ-15 was positively correlated with sleep disturbances $(r=0.38$, $p<0.05)$, use of sleeping medication $(r=0.56, p<0.05)$, and daytime dysfunction $(r=0.56, p<0.05)$ of PSQI. In the GCBT group, the score change in worrying about sleep of DBAS-16 
Table 2 Mean scale scores (mean \pm SD) after 16 weeks (in parentheses) and their changes (baseline minus conditioned, immediately after parentheses) in the PHQ-9, PHQ-I5, DBAS-16, and PSQI questionnaires from the GCBT ( $n=26)$, zolpidem ( $n=27)$, and healthy control $(n=3 I)$ groups

\begin{tabular}{|c|c|c|c|}
\hline Scale & GCBT & Zolpidem & Healthy controls \\
\hline PHQ-9 & $(4.58 \pm 4.69) 2.73 \pm 4.95^{\mathrm{a}}$ & $(3.93 \pm 3.56) 2.48 \pm 5.87^{a}$ & $(0.94 \pm 1.53) 0.10 \pm 0.54$ \\
\hline PHQ-I5 & $(3.88 \pm 3.74)|.85 \pm 3.4|^{a}$ & (4.07 \pm 4.04$) 0.70 \pm 3.36$ & 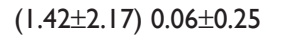 \\
\hline \multicolumn{4}{|l|}{ DBAS- 16} \\
\hline Consequence of insomnia & $(\mid 4.8 \mathrm{I} \pm 4.23) 3.62 \pm 4.46^{\mathrm{a}, \mathrm{b}}$ & 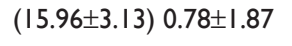 & $(14.62 \pm 3.74)-0.06 \pm 0.57$ \\
\hline Worrying about sleep & $(17.62 \pm 4.56) 4.85 \pm 5.56^{\mathrm{a}, \mathrm{b}}$ & $(19.22 \pm 3.38) 2.22 \pm 3.53^{\mathrm{a}}$ & $(15.37 \pm 5.45)-0.06 \pm 0.63$ \\
\hline Sleep expectations & $(6.0 \mathrm{I} \pm 2.08) \mathrm{I} .8 \mathrm{I} \pm 2.37^{\mathrm{a}, \mathrm{b}}$ & $(6.57 \pm 2.13) 0.04 \pm 1.32$ & $(4.22 \pm 2.78) 0.23 \pm 1.67$ \\
\hline Medication attribution & $(7.65 \pm I .8 \mathrm{I}) 1.00 \pm 2.17^{\mathrm{a}, \mathrm{b}}$ & $(8.56 \pm 1.67)-0.56 \pm 1.87$ & $(7.68 \pm 3.54) 0.19 \pm 0.70$ \\
\hline \multicolumn{4}{|l|}{ PSQI } \\
\hline Subjective sleep quality & $(1.04 \pm 0.72) 0.73 \pm 0.92^{\mathrm{a}}$ & $(1.22 \pm 0.58) 0.45 \pm 0.89^{a}$ & $(0.39 \pm 0.50) 0.00 \pm 0.00$ \\
\hline Sleep latency & $(1.46 \pm 0.95) 0.42 \pm 0.90^{\mathrm{a}}$ & 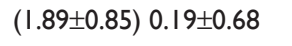 & $0.7 I \pm 0.74) 0.00 \pm 0.00$ \\
\hline Sleep duration & (I.I5 $\pm I .0 I) 0.23 \pm 0.99$ & (I.33 $\pm I .07) 0.07 \pm I .00$ & $(0.29 \pm 0.53) 0.00 \pm 0.26$ \\
\hline Habitual sleep efficiency & $0.69 \pm 1.05) 0.92 \pm 1.29^{a, b}$ & $(I .37 \pm I .2 I) 0.30 \pm I .20$ & $(0.13 \pm 0.43) 0.00 \pm 0.00$ \\
\hline Sleep disturbances & (I.3I \pm 0.55$) 0.50 \pm 0.8 \mathrm{I}^{\mathrm{a}, \mathrm{b}}$ & $(1.48 \pm 0.58) 0.15 \pm 0.60$ & $(0.94 \pm 0.44) 0.00 \pm 0.00$ \\
\hline Sleeping medication use & (I.73 \pm 1.43$) 0.77 \pm 1.42^{\mathrm{a}, \mathrm{b}}$ & $(2.48 \pm 1.12)-0.07 \pm 1.04$ & $(0.13 \pm 0.34) 0.00 \pm 0.00$ \\
\hline Daytime dysfunction & 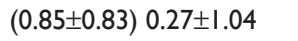 & $0.48 \pm 0.89) 0.33 \pm 1.00$ & $(0.42 \pm 0.62) 0.03 \pm 0.18$ \\
\hline
\end{tabular}

Notes: ${ }^{a} p<0.05$ vs healthy control group. ${ }^{b} p<0.05$ vs zolpidem group. Data presented as mean \pm SD.

Abbreviations: DBAS-16, Dysfunctional Beliefs and Attitudes about Sleep-16; GCBT, group cognitive-behavioral therapy; PHQ-9, Patient Health Questionnaire-9; PHQ-15, Patient Health Questionnaire-15; PSQI, Pittsburgh Sleep Quality Index.

was positively correlated with that of subjective sleep quality of PSQI ( $\mathrm{n}=26, r=0.41, p<0.05)$, and the score change in medication attribution of DBAS-16 was positively correlated with that of daytime dysfunction of PSQI $(r=0.44, p<0.05)$.

\section{Discussion}

Zolpidem treatment helped our GCBT patients receive psychoeducation during the first 4 weeks, and as a medication control, its effect contrasted to that of GCBT for the remaining 12 weeks. For instance, insomnia patients have shown significant scale score decrements of PHQ-9, PHQ-15, DBAS-16, and PSQI after GCBT. Our results were consistent with the documentation that $\mathrm{CBT}$ intervention played a role in altering the dysfunctional beliefs and attitudes about sleep, improving the subjective sleep quality, as well as lowering the somatic and emotional problems in patients. ${ }^{28-30}$

Previous results have shown that poor sleep was linked with depressive mood and somatic symptoms, ${ }^{14,31}$ which supports our finding that insomnia patients scored higher in PHQ-9 and PHQ-15 in the current study. Our patients in the GCBT group displayed significant reductions in these scales after intervention, which was also consistent with the suggestion that the sleep improvement enhanced physical and mental health conditions. ${ }^{13,14}$ Other investigations have shown that better sleep leads to improvements in emotional processing, affect normalizing, ${ }^{32,33}$ as well as leads to improvements pain management, ${ }^{13}$ which also agrees with the score reduction after GCBT in our study.
The insomnia patients endorsed stronger dysfunctional beliefs and attitudes about sleep than the self-defined good sleepers, ${ }^{34-36}$ as the higher DBAS-16 scale scores were found in our patients. It has been hypothesized that when insomniacs hold strongly unrealistic expectations about sleep, they tend to worry excessively when such requirements are not met. ${ }^{37}$ The GCBT applied in the current study helped to restructure the sleep cognitions and reshape the sleep-related behaviors to alter the dysfunctional beliefs and attitudes about sleep in patients. Previous results have shown that when the unrealistic expectations were lowered, the level of worrying about sleep was normalized. ${ }^{36}$ Similarly, the use of medication was reduced in patients as well; our current findings were thus in line with these reports.

Patients with insomnia scored higher on almost all PSQI scales, which was consistent with previous psychotherapy studies. ${ }^{18-38}$ After GCBT, the subjective sleep quality of PSQI was significantly improved in patients, which was consistent with previous reports. ${ }^{29-39}$ Consequently, the sleep improvement experience might contribute to the reduction in sleep disturbances and to less dependence on hypnotics; thus, our patients reported improved habitual sleep efficacy and less sleeping medication usage after GCBT. Regarding the aspects of sleep latency and habitual sleep efficiency of PSQI, their improvements after GCBT in our study were also in line with the previous notion of the psychotherapy effect for insomnia. ${ }^{40}$ 
In our study, the correlations found between the scale score changes in PSQI and other inventories in the zolpidem group were in line with those reports showing that the depressive mood, somatic symptoms, and the altered beliefs and attitudes contributed to poor sleep. ${ }^{3,35,41}$ After GCBT, the cognitive components about sleep were restructured and the mood and somatic functions were normalized; the significant correlations were thus confined to the two between DBAS-16 and PSQI scales. The outcomes still pointed to the importance of belief and attitude improvements in sleep recovery, as demonstrated previously. ${ }^{29,37,40}$

One should also notice the limitations of our study design. First, our study does not belong to a typical longitudinal design; the inventory assessments were only taken at two time points. It remains unknown whether the GCBT effect would last for a longer period. Second, there are other effective ways to measure psychological or somatic disturbances in insomnia besides the PHQ-9 and PHQ-15. For instance, the psychopathology of insomnia is the presleep cognitive and somatic hyperarousal levels, ${ }^{42}$ questionnaires such as the Pre-sleep Arousal Scale ${ }^{43}$ might be more relevant. Third, our study lacks control groups such as the waiting-list (or sham psychotherapy) patients or those treated with both zolpidem and GCBT; the current psychotherapeutic effect needs further confirmation. Fourth, we did not measure the personality traits of our participants, which might influence the current results. Fifth, the GCBT group had received 4 weeks of zolpidem; whether the medication had influenced the later-on effect of GCBT was unknown. Nevertheless, we have demonstrated the GCBT effectiveness in insomnia, which supports the advocacy of applying CBT to sleep and related disorders. ${ }^{16,17,30}$

\section{Acknowledgments}

WW was sponsored by a grant from the Natural Science Foundation of China (No 91132715).

\section{Disclosure}

The authors report no conflicts of interest in this work.

\section{References}

1. Ohayon MM. Epidemiology of insomnia: what we know and what we still need to learn. Sleep Med Rev. 2002;6(2):97-111.

2. Morin CM, Bélanger L, LeBlanc M, et al. The natural history of insomnia: a population-based 3-year longitudinal study. Arch Intern Med. 2009; 169(5):447-453.

3. Johnson EO, Roth T, Breslau N. The association of insomnia with anxiety disorders and depression: exploration of the direction of risk. J Psychiatr Res. 2006;40(8):700-708.

4. Baglioni C, Battagliese G, Feige B, et al. Insomnia as a predictor of depression: a meta-analytic evaluation of longitudinal epidemiological studies. J Affect Disord. 2009;135(1-3):10-19.

5. Anderson KN, Bradley AJ. Sleep disturbance in mental health problems and neurodegenerative disease. Nat Sci Sleep. 2013;5:61-75.
6. Morgan K, Clarke D. Longitudinal trends in late-life insomnia: implications for prescribing. Age Ageing. 1997;26(3):179-184.

7. Riemann D, Perlis ML. The treatments of chronic insomnia: a review of benzodiazepine receptor agonists and psychological and behavioral therapies. Sleep Med Rev. 2009;13(3):205-214.

8. Ohayon MM, Caulet M, Priest RG, Guilleminault C. Psychotropic medication consumption patterns in the UK general population. J Clin Epidemiol. 1998;51(3):273-283.

9. Kramer M. Hypnotic medication in the treatment of chronic insomnia: non nocere! Doesn't anyone care? Sleep Med Rev. 2000;4(6):529-541.

10. Espie CA. Insomnia: conceptual issues in the development, persistence, and treatment of sleep disorder in adults. Annu Rev Psychol. 2002;53(1):215.

11. Morin CM, Stone J, Trinkle D, Mercer J, Remsberg S. Dysfunctional beliefs and attitudes about sleep among older adults with and without insomnia complaints. Psychol Aging. 1993;8(3):463-467.

12. Morin CM, Culbert JP, Schwartz SM. Nonpharmacological interventions for insomnia: a meta-analysis of treatment efficacy. $\mathrm{Am} \mathrm{J}$ Psychiatry. 1994;151(8):1172-1180.

13. Vitiello MV, Rybarczyk B, Von Korff M, Stepanski EJ. Cognitive behavioral therapy for insomnia improves sleep and decreases pain in older adults with co-morbid insomnia and osteoarthritis. J Clin Sleep Med. 2009;5(4):355-362.

14. Manber R, Bernert RA, Suh S, Nowakowski S, Siebern AT, Ong JC. CBT for insomnia in patients with high and low depressive symptom severity: adherence and clinical outcomes. J Clin Sleep Med. 2011;7(6):645-652.

15. Ulmer CS, Edinger JD, Calhoun PS. A multi-component cognitivebehavioral intervention for sleep disturbance in veterans with PTSD: a pilot study. J Clin Sleep Med. 2011;7(1):57-68.

16. Espie CA. 'Stepped care': a health technology solution for delivering cognitive behavioral therapy as a first line insomnia treatment. Sleep. 2009;32(12):1549-1558.

17. Edinger JD. Is it time to step up to stepped care with our cognitivebehavioral insomnia therapies? Sleep. 2009;32(12):1539.

18. Koffel EA, Koffel JB, Gehrman PR. A meta-analysis of group cognitive behavioral therapy for insomnia. Sleep Med Rev. 2015;19:6-16.

19. Ritterband LM, Thorndike FP, Ingersoll KS, et al. Effect of a webbased cognitive behavior therapy for insomnia intervention with 1-year follow-up: a randomized clinical trial. JAMA Psychiatry. 2017; 74(1):68-75.

20. Krystal AD, Prather AA. Should internet cognitive behavioral therapy for insomnia be the primary treatment option for insomnia? Toward Getting More SHUTi. JAMA Psychiatry. 2017;74(1):15-16.

21. Sirdifield C, Anthierens S, Creupelandt H, Chipchase SY, Christiaens T, Siriwardena AN. General practitioners' experiences and perceptions of benzodiazepine prescribing: systematic review and meta-synthesis. BMC Fam Pract. 2013;14:191.

22. Morin CM. Insomnia: Psychological Assessment and Management. New York, US: Guilford Press; 1993.

23. Morin CM, Vallières A, Ivers H. Dysfunctional beliefs and attitudes about sleep (DBAS): validation of a brief version (DBAS-16). Sleep. 2007; 30(11):1547-1554.

24. Buysse DJ, Reynolds CF, Monk TH, Berman SR, Kupfer DJ. The Pittsburgh Subjective Sleep Quality Index: a new instrument for psychiatric practice and research. Psychiatry Res. 1989;28(2):193-213.

25. Kroenke K, Spitzer RL, Williams JB. The PHQ-9: validity of a brief depression severity measure. J Gen Intern Med. 2001;16(9):606-613.

26. Kroenke K, Spitzer RL, Williams JB. The PHQ-15: validity of a new measure for evaluating the severity of somatic symptoms. Psychosom Med. 2002;64(2):258-266.

27. American Psychiatric Association. Diagnostic and Statistical Manual of Mental Disorder. 5th ed. Arlington, VA: American Psychiatric Publishing; 2013.

28. Morin CM, Hauri PJ, Espie CA, Spielman AJ, Buysse DJ, Bootzin RR. Nonpharmacologic treatment of chronic insomnia. An American Academy of Sleep Medicine review. Sleep. 1999;22(8):1134-1156. 
29. Espie CA, Inglis SJ, Tessier S, Harvey L. The clinical effectiveness of cognitive behaviour therapy for chronic insomnia: implementation and evaluation of a sleep clinic in general medical practice. Behav Res Ther. 2001;39(1):45-60.

30. Miller CB, Espie CA, Kyle SD. Cognitive behavioral therapy for the management of poor sleep in insomnia disorder. Chronophysiol Ther. 2014;9:11

31. Wagley JN, Rybarczyk B, Nay WT, Danish S, Lund HG. Effectiveness of abbreviated CBT for insomnia in psychiatric outpatients: sleep and depression outcomes. J Clin Psychol. 2013;69(10):1043-1055.

32. Harvey AG. Insomnia, psychiatric disorders, and the transdiagnostic perspective. Curr Dir Psychol Sci. 2008;17(5):299-303.

33. Walker MP, van der Helm E. Overnight therapy? The role of sleep in emotional brain processing. Psychol Bull. 2009;135(5):731-748.

34. Edinger JD, Fins AI, Glenn DM, et al. Insomnia and the eye of the beholder: are there clinical markers of objective sleep disturbances among adults with and without insomnia complaints? J Consult Clin Psychol. 2000;68(4):586-593.

35. Carney CE, Edinger JD, Morin CM, et al. Examining maladaptive beliefs about sleep across insomnia patient groups. J Psychosom Res. 2010; 68(1):57-65.

36. Montserrat Sánchez-Ortuño M, Edinger JD. A penny for your thoughts: patterns of sleep-related beliefs, insomnia symptoms and treatment outcome. Behav Res Ther. 2001;48(2):125-133.
37. Morin CM, Blais F, Savard J. Are changes in beliefs and attitudes about sleep related to sleep improvements in the treatment of insomnia? Behav Res Ther. 2002;40(7):741-752.

38. Smith MT, Perlis ML, Park A, et al. Comparative meta-analysis of pharmacotherapy and behavior therapy for persistent insomnia. Am J Psychiatry. 2002;159(1):5-10.

39. Murtagh DR, Greenwood KM. Identifying effective psychological treatments for insomnia: a meta-analysis. J Consult Clin Psychol. 1995; 63(1):79-89

40. Okajima I, Komada Y, Inoue Y. A meta-analysis on the treatment effectiveness of cognitive behavioral therapy for primary insomnia. Sleep Biol Rhythms. 2011;9(1):24-34.

41. Espie CA, Inglis SJ, Harvey L, Tessier S. Insomniacs' attributions: psychometric properties of the Dysfunctional Beliefs and Attitudes about Sleep Scale and the Sleep Disturbance Questionnaire. J Psychosom Res. 2000;48(2):141-148.

42. American Academy of Sleep Medicine. The International Classification of Sleep Disorders. 3rd ed. Darien, IL: American Academy of Sleep Medicine; 2014.

43. Nicassio PM, Mendlowitz DR, Fussell JJ, Petras L. The phenomenology of the pre-sleep state: the development of the pre-sleep arousal scale. Behav Res Ther. 1985;23(3):263-271. 


\section{Supplementary material}

Table SI Summary of the manual of group cognitive-behavioral therapy

\begin{tabular}{|c|c|c|}
\hline Course and theme & Therapist's role & Patient's role \\
\hline I. Insomnia self-management (I) & $\begin{array}{l}\text { TI.I. Demonstrate the role of self-management and } \\
\text { the power of teamwork } \\
\text { TI.2. Guide patients to establish a team contract }\end{array}$ & $\begin{array}{l}\text { PI.I. Introduce self } \\
\text { PI.2. Join a team by a contract } \\
\text { PI.3. Set goals for the following } 2 \text { weeks }\end{array}$ \\
\hline 2. Insomnia self-management (II) & $\begin{array}{l}\text { T2.I. Demonstrate the role and the meaning of } \\
\text { positive action } \\
\text { T2.2. Deliver techniques and skills of sleep self- } \\
\text { monitoring and time management }\end{array}$ & $\begin{array}{l}\text { P2.I. Feedback what has been accomplished } \\
\text { according to goals set during the last course } \\
\text { P2.2. Set goals for the following } 2 \text { weeks }\end{array}$ \\
\hline 3. Misunderstanding of insomnia & $\begin{array}{l}\text { T3.I. Evaluate the therapy effect of the previous } \\
4 \text { weeks } \\
\text { T3.2. Deliver the knowledge of sleep hygiene and } \\
\text { sleep energy } \\
\text { T3.3. Demonstrate the effects of medication/drugs } \\
\text { and alcohol on sleep }\end{array}$ & $\begin{array}{l}\text { P3.I. Feedback what has been accomplished } \\
\text { according to goals set during the last course } \\
\text { P3.2. Brainstorm for realizing some } \\
\text { misunderstandings of insomnia } \\
\text { P3.3. Set goals for the following } 2 \text { weeks }\end{array}$ \\
\hline 4. Positive behavior training & $\begin{array}{l}\text { T4.I. Demonstrate roles of aerobic exercise and } \\
\text { positive interpersonal communication and interests } \\
\text { on sleep }\end{array}$ & $\begin{array}{l}\text { P4.I. Feedback what has been accomplished } \\
\text { according to goals set during last course } \\
\text { P4.2. Set goals for the following } 2 \text { weeks }\end{array}$ \\
\hline 5. Relaxation therapy & $\begin{array}{l}\text { T5.I. Demonstrate the role of relaxation } \\
\text { T5.2. Guide patients doing relaxation training }\end{array}$ & $\begin{array}{l}\text { P5.I. Feedback what has been accomplished } \\
\text { according to goals set during the last course } \\
\text { P5.2. Do some relaxation training, eg, abdominal } \\
\text { respiration, progressive muscle relaxation, and } \\
\text { guided imagination } \\
\text { P5.3. Set goals for the following } 2 \text { weeks }\end{array}$ \\
\hline 6. Cognitive restructuring & $\begin{array}{l}\text { T6.I. Deliver techniques of distinguishing thought } \\
\text { traps } \\
\text { T6.2. Deliver techniques of how to operate } \\
\text { programmed theoretical knowledge }\end{array}$ & $\begin{array}{l}\text { P6.1. Feedback what has been accomplished } \\
\text { according to goals set during the last course } \\
\text { P6.2. Discuss techniques to minimize negative } \\
\text { thoughts } \\
\text { P6.3. Set goals for the following } 2 \text { weeks }\end{array}$ \\
\hline 7. Physical treatment & $\begin{array}{l}\text { T7.I. Feedback through electroencephalogram } \\
\text { T7.2. Feedback with visible music }\end{array}$ & $\begin{array}{l}\text { P7.I. Feedback what has been accomplished } \\
\text { according to goals set during the last course } \\
\text { P7.2. Set goals for the following } 2 \text { weeks }\end{array}$ \\
\hline $\begin{array}{l}\text { 8. Sleep self-efficiency } \\
\text { improvement }\end{array}$ & $\begin{array}{l}\text { T8.I. Emphasize the importance of the repeated } \\
\text { therapy } \\
\text { T8.2. Deliver techniques to prevent insomnia relapse }\end{array}$ & $\begin{array}{l}\text { P8. I. Feedback what has been accomplished } \\
\text { according to goals set during the last course } \\
\text { P8.2. Get in touch with team members in the future }\end{array}$ \\
\hline
\end{tabular}

Notes: Each session lasted for 2 weeks; sleep hygiene practices included diet, exercise, and environmental preparations.

\section{Publish your work in this journal}

Neuropsychiatric Disease and Treatment is an international, peerreviewed journal of clinical therapeutics and pharmacology focusing on concise rapid reporting of clinical or pre-clinical studies on a range of neuropsychiatric and neurological disorders. This journal is indexed on PubMed Central, the 'PsycINFO' database and CAS, and is the official journal of The International Neuropsychiatric Association (INA). The manuscript management system is completely online and includes a very quick and fair peer-review system, which is all easy to use. Visit http://www.dovepress.com/testimonials.php to read real quotes from published authors. 